\title{
Precise RFID Localization in Impaired Environment through Sparse Signal Recovery
}

\author{
Saurav Subedi, Yimin D. Zhang, and Moeness G. Amin \\ Center for Advanced Communications, Villanova University, Villanova, PA 19085
}

\begin{abstract}
Radio frequency identification (RFID) is a rapidly developing wireless communication technology for electronically identifying, locating, and tracking products, assets, and personnel. RFID has become one of the most important means to construct real-time locating systems (RTLS) that track and identify the location of objects in real time using simple, inexpensive tags and readers. The applicability and usefulness of RTLS techniques depend on their achievable accuracy. In particular, when multilateration-based localization techniques are exploited, the achievable accuracy primarily relies on the precision of the range estimates between a reader and the tags. Such range information can be obtained by using the received signal strength indicator (RSSI) and/or the phase difference of arrival (PDOA). In both cases, however, the accuracy is significantly compromised when the operation environment is impaired. In particular, multipath propagation significantly affects the measurement accuracy of both RSSI and phase information. In addition, because RFID systems are typically operated in short distances, RSSI and phase measurements are also coupled with the reader and tag antenna patterns, making accurate RFID localization very complicated and challenging. In this paper, we develop new methods to localize RFID tags or readers by exploiting sparse signal recovery techniques. The proposed method allows the channel environment and antenna patterns to be taken into account and be properly compensated at a low computational cost. As such, the proposed technique yields superior performance in challenging operation environments with the above-mentioned impairments.
\end{abstract}

\section{INTRODUCTION}

Radio frequency identification (RFID) is a widely used technology for electronic identification, localization and tracking of products, assets, and personnel. Some important applications include logistics, transportations, health-care sectors, access control, pharmaceutical industry and so forth $[1,2]$. Desired degree of accuracy in RFID localization may vary among different applications. Precise localization of RFID tags or readers is imperative, especially for real-time locating systems (RTLS) applications.

An RFID positioning system constitutes of two major functional blocks, i.e., location sensing and positioning processing [3]. Location sensing involves sensing the location of RFID element (reader or tag) in terms of range and/or direction-of-arrival (DOA) using proper location metrics. Range estimates may be obtained through any of these prevailing methods - received signal strength indication (RSSI) measurements [4], round trip-of-flight (TOF) [5], time-difference-of-arrival (TDOA) [6], and/or phase-difference-of-arrival (PDOA) measurements [7,8] of RFID signals. DOA information is typically acquired using directional antenna, phased array, and/or smart antenna $[3,9]$. The information about range, DOA, and/or the propagation characteristics is then exploited in the positioning processing methods.

RFID positioning algorithms have been classified into five categories [3], namely, trilateration/multilateration, triangulation, hybrid direction/range method, radio map matching method, and constraint based approach. Trilateration/multilateration technique [10] utilizes the range information estimated at several spatially separated reference points, whereas triangulation method [1] is based on DOA of received signal from two or more reference points. Hybrid direction/range method is applicable when both DOA and range information are available [11]. Radio map matching or scene analysis is a two-step process that involves the deployment of extra reference tags. First, propagation environment is assessed using the reference tags, yielding a set of fingerprints. Then, the actual measurements are compared against the fingerprints to estimate the target's location [12]. Constraint-based approach [13] is based on connectivity information, by defining inclusive constraints (tags that are within the coverage range of the reader) and exclusive constraints (tags that are out of the reader's coverage range). A survey of the state-of-the art related to RFID localization is presented in [14] along with the classification of different 


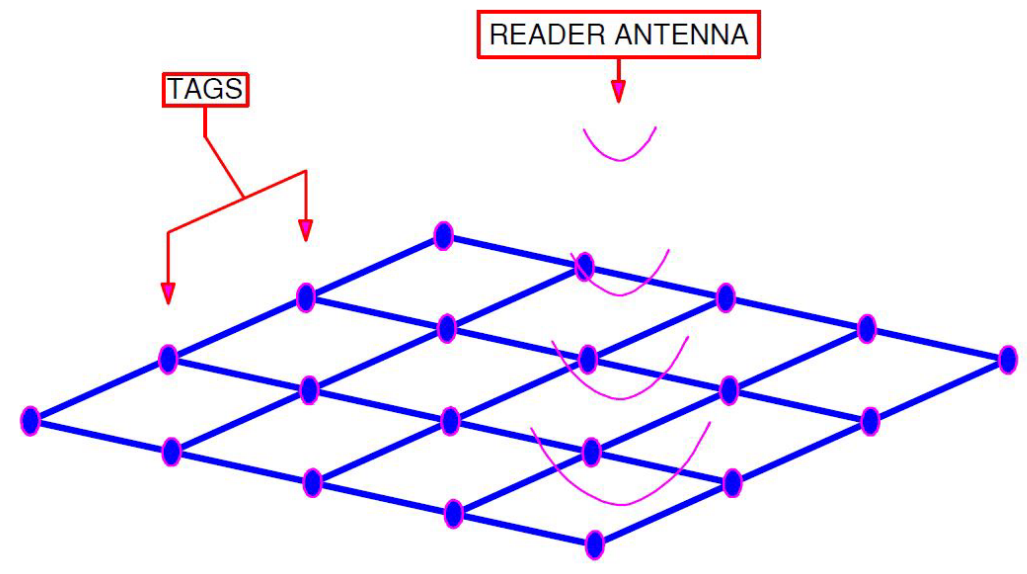

Figure 1. Schematics of a typical RFID reader-tags arrangement

localization methods and algorithms. Selection of appropriate sensing and positioning technique is influenced by the resource constraints (e.g. bandwidth and power limitations), propagation conditions (e.g. indoor, outdoor), and system limitations. In complex RF propagation environment, typically characterized by line-of-sight (LOS) obstructions, and reflections and scattering, signal undergo severe attenuation due to shadowing and multipath causes a rapid fluctuation in signal strength even within a fraction of a wavelength. This adversely affects the accuracy of information, both range and DOA, acquired from the location sensing algorithms. This error propagates through to the positioning methods resulting in inaccurate location estimation.

RSSI-based range estimation methods are widely used on account of their simplicity and effectiveness. However, like other location sensing methods, the range estimation performance from RSSI, is not robust in impaired propagation conditions. In presence of multitude of propagation paths, RSSI is not related monotonically with the range, causing an ambiguity [15]. Directional antenna pattern is another factor that renders significant effects particularly in the near field measurements [16]. These two propagation phenomenon are typical to RFID localization problems and may considerably effect the achievable accuracy in location estimation. Therefore, it is important to devise a mechanism to compensate for these propagation impairments such that a monotonic relationship between range and RSSI can be formulated.

In this paper, we consider the problem of precisely estimating position an RFID reader in a passive RFID deployment in an impaired propagation environment characterized by multipath. We also consider the effect of non-isotropic antenna radiation pattern on localization performance. We separately analyze the effects of these two propagation impairments on the localization performance. RSSI measurement is used for range estimation and reader position is estimated by multilaterating the range information obtained from multiple tags at known positions. With the a priori knowledge that the problem considers localization of a single reader, we exploit sparse signal recovery based method, which allows us to compensate for the effects of multipath and antenna directivity. Simulation results show that the proposed method effectively compensates for the effect of propagation impairments and achieves a precise estimation of the reader position.

Notations: We use lower-case (upper-case) bold characters to denote vectors (matrices). $(.)^{T}$ denotes the transpose of a matrix or vector, $\|\cdot\|_{0},\|\cdot\|_{1}$ and $\|\cdot\|_{2}$, respectively, denote the $l_{0}, l_{1}$ and $l_{2}$ norm of a vector.

\section{RSSI-BASED RFID READER LOCALIZATION THROUGH MULTILATERATION}

In a typical passive RFID system, as depicted in Figure 1, signal transmitted from a reader is backscattered by tags such that the signal attenuates along the round-trip back to the reader. According to the Friis transmission equation, the signal power received at the reader due to the signal backscattered from the $i$ th tag is expressed as $[17]$

$$
P_{i, \mathrm{Rx}}=P_{\mathrm{Tx}} G_{\mathrm{tag}}^{2} G_{\mathrm{reader}}^{2}\left(\frac{\lambda}{4 \pi d_{i}}\right)^{2 \eta}
$$


where $P_{\mathrm{Tx}}$ is the transmit power from the reader, $G_{\text {tag }}$ and $G_{\text {reader }}$, respectively, are the directional gains of tag and reader antennas, $\lambda$ is the wavelength, and $d_{i}$ is the range between the $i$ th tag and the reader. The path loss exponent, $\eta$, varies depending upon the propagation environment. Considering that the reader has line-of-sight with the tags, free space propagation is assumed, i.e., $\eta$ is considered equal to 2 . Therefore, the range between the reader and the $i$ th tag can be calculated from the RSSI measurements as follows,

$$
d_{i}=\frac{\lambda}{4 \pi}\left(\frac{P_{\mathrm{Tx}} G_{\mathrm{tag}}^{2} G_{\mathrm{reader}}^{2}}{P_{i, \mathrm{Rx}}}\right)^{\frac{1}{4}} .
$$

These range estimates can be used to localize the reader using the multilateration method. Range information from at least $n+1$ tags is required to unambiguously localize the reader in the $n$-dimensional space. Therefore, the location of the reader in the three-dimensional (3-D) space, $(x, y, z)$, can be determined by

$$
\left(x-x_{i}\right)^{2}+\left(y-y_{i}\right)^{2}+\left(z-z_{i}\right)^{2}=d_{i}^{2}, \quad i=1,2, \ldots, N,
$$

where $N \geq n+1$. In this paper, the tags are assumed to be located on the floor, i.e., $z_{i}=0$, and the reader is assumed to be at a known constant height, $h$. As such, the underlying problem can be analyzed in two dimensions. Therefore, at least three range estimates $(N \geq 3)$ are required to localize the reader in the two dimensional (2-D) space with unknown reader position determined by its $x$ - and $y$ - axis coordinates. In this case, equation (3) can be modified as

$$
\left(x-x_{i}\right)^{2}+\left(y-y_{i}\right)^{2}+h^{2}=d_{i}^{2}, \quad i=1,2, \ldots, N .
$$

In matrix-vector representation, equation (4) can be written as [3]

$$
\mathbf{A}\left(\begin{array}{l}
x \\
y
\end{array}\right)=\mathbf{b}
$$

where

$$
\mathbf{A}=\left(\begin{array}{cc}
x_{1}-x_{2} & y_{1}-y_{2} \\
\vdots & \vdots \\
x_{N-1}-x_{N} & y_{N-1}-y_{N}
\end{array}\right)
$$

is an $(N-1) \times 2$ matrix and

$$
\mathbf{b}=\frac{1}{2}\left(\begin{array}{c}
d_{2}^{2}-d_{1}^{2}+x_{1}^{2}-x_{2}^{2}+y_{1}^{2}-y_{2}^{2} \\
\vdots \\
d_{M}^{2}-d_{M-1}^{2}+x_{M-1}^{2}-x_{M}^{2}+y_{M-1}^{2}-y_{M}^{2}
\end{array}\right)
$$

is an $(N-1) \times 1$ vector. Assuming that $N>3$ and the tags are not collinearly located, the $(x, y)$ coordinates of the RFID reader are obtained as the least-square solution of equation (5), given by

$$
\left(\begin{array}{l}
x \\
y
\end{array}\right)=\left(\mathbf{A}^{T} \mathbf{A}\right)^{-1} \mathbf{A}^{T} \mathbf{b} .
$$

RSSI-based localization methods, thus, rely on the range estimates to localize the RFID reader. Equations (1) and (2) reveal that the RSSI measurements and, consequently, the range estimates, depend on the antenna radiation patterns and the distance between a tag and the reader. Since RFID systems are typically operated over short distances, the RSSI measurements are coupled with the reader and the tag antenna patterns. Therefore, antenna patterns significantly affect the RSSI measurements. The range estimates, therefore, can be ambiguous in presence of non-isotropic radiation patterns. Multipath propagation is another major source of error in traditional RSSI based localization methods. The RF signals propagating along different paths are linearly combined at the RFID reader, constructively or destructively, depending on their relative phases. This causes significant variations in signal strength even within a fraction of a wavelength, which adds to the ambiguity in range estimates calculated on the basis of RSSI measurements. In the following, we propose a new method that allows the channel environment and antenna patterns to be taken into account and be properly compensated at a low computational cost. 


\section{PRECISE RFID READER LOCALIZATION}

In this section, we separately analyze the effects of two important propagation factors, namely, antenna radiation pattern and multipath propagation, on the accuracy of localization. Sparsity based signal recovery method is used to compensate for these propagation impairments. Simulation results, presented in the following section, verify that through compensation, we are able to achieve higher precision in RFID reader positioning.

\section{A. Precise RFID Reader Localization with Reader Antenna Directivity}

Conventionally, RSSI-based localization algorithms assume isotropic radiation patterns for the reader and the tag antennas [18]. However, in practice, the effective radiation pattern is non-isotropic; consequently, the range estimates are biased towards the direction of high gain in the radiation pattern. In this paper, we only consider the effect of reader antenna patterns. The tag pattern can be made orientation-independent through the use of two orthogonal tags (called "Super" RFID tags [19]) such that the sum power from the two tags is almost isotropic. The effect of reader antenna pattern is negligible in the far-field. However, in the near-field case, the antenna gain depends on the relative position between the reader and the tag and, as such, the spatial directivity has a pronounced effect on the RSSI measurements [16]. Higher RSS is obtained, even at the same propagation range, towards the direction of high antenna gain. Consequently, the range estimations calculated using equation (2) are inconclusive. Therefore, it is desirable to compensate for the effect of directional radiation pattern on RSSI measurements, and subsequently on range estimation.

With the a priori information that there are sparsely located readers in operation, the underlying problem can be solved using sparse signal recovery method. In this paper, we consider localization of a single reader from the RSSI measurements obtained from multiple tags at known locations within the search area. The entire search area is modeled as an $N_{x} \times N_{y}$ rectangular grid and, as such, each point in the grid represents a hypothetical reader position. Let $\boldsymbol{\Phi}$ of dimension $N \times N_{x} N_{y}$ be the dictionary matrix representing the search grid, whose $k$ th column is expressed as

$$
\phi_{k}=\left[\phi_{1}^{k}, \phi_{2}^{k}, \ldots, \phi_{N}^{k}\right]^{T},
$$

where $k=1, \ldots, N_{x} N_{y}$. Element $\phi_{i}^{k}$ represents the RSSI measurement corresponding to the $k$ th hypothetical reader position at $\left(x_{k}, y_{k}, h\right)$ and the $i$ th tag located at $\left(x_{i}, y_{i}, 0\right)$ and are calculated as

$$
\phi_{i}^{k}=P_{\mathrm{Tx}} G_{\mathrm{tag}}^{2} G_{\text {reader }}^{2}\left(\frac{\lambda}{4 \pi d_{i}^{k}}\right)^{4}
$$

where

$$
d_{i}^{k}=\sqrt{\left(x_{k}-x_{i}\right)^{2}+\left(y_{k}-y_{i}\right)^{2}+h^{2}} .
$$

Define $\mathbf{y}$ as the $N$-element vector which stacks the RSSI measurements at the actual reader position corresponding to the $N$ tags. It is noted that, out of the $N$ tags, only a subset of tags receive sufficient energy to respond back to the reader for valid RSSI measurements. These non-responsive tags should be removed from evaluation. An $N_{x} N_{y} \times 1$ vector $\mathbf{u}$ with unknown and sparse entries, which vectorizes the discretized 2-D spatial indices, is to be estimated, such that the $k$ th entry of $\mathbf{u}$ is associated with the 2-D reader position $\left(x_{k}, y_{k}\right)$. Rewriting the problem in a matrix-vector form, we have

$$
\mathbf{y}=\mathbf{\Phi} \mathbf{u}
$$

For high accuracy reader localization, this is an underdetermined system of linear equations as the observation vector $\mathbf{y}$ is $N \times 1$, whereas the estimation vector $\mathbf{u}$ is of dimension $N_{x} N_{y} \times 1$, where $N_{x} N_{y} \gg N$. For single entry vector $\mathbf{u}$, this can be optimally solved using the standard maximum likelihood (ML) search as

$$
\hat{\mathbf{u}}_{k}=\arg \min _{k}\left\|\mathbf{y}-\phi_{k} u_{k}\right\|^{2},
$$

where $\phi_{k}$ is the $k$ th column of the dictionary matrix $\boldsymbol{\Phi}$ that exhibits maximum correlation to the actual RSSI measurements and $u_{k}$ is the corresponding non-zero element in the sparse vector $\mathbf{u}$. 


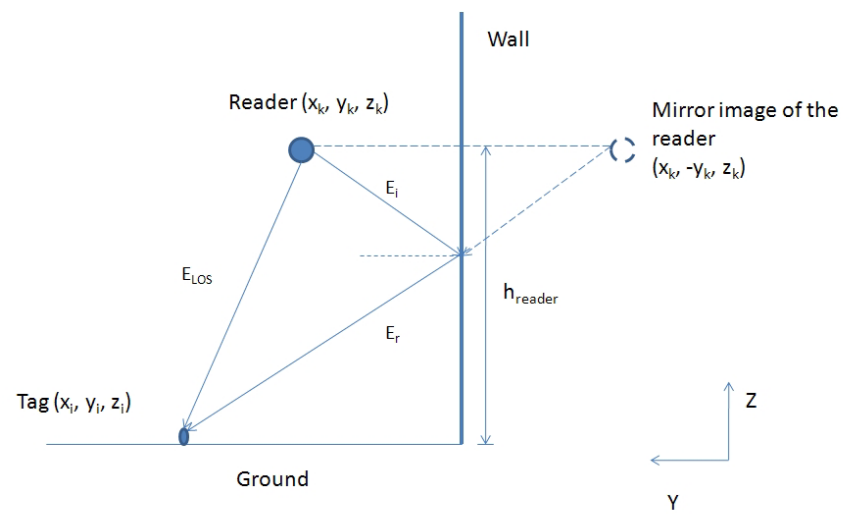

Figure 2. Multipath Propagation

The proposed method becomes computationally exhaustive when multiple readers are involved to energize the tags, such that a multidimensional search is required. In order to improve the computational efficiency of the proposed algorithm and extend its applicability in such situations, we can apply sparsity based methods to solve the underlying problem. By adding a constraint of sparsity, i.e., to minimize the number of non-zero elements in the solution, this problem can be reformulated as a standard $l_{0}$-norm minimization problem, such that

$$
\min \|\mathbf{u}\|_{0} \quad \text { subject to } \quad \mathbf{y}=\boldsymbol{\Phi} \mathbf{u} .
$$

Mathematically, the $l_{0}$-norm minimization minimizes the number of non-zero elements in the solution and, therefore, gives an $n$-sparse solution corresponding to the $n$ reader locations. To reduce the computation cost, sparsity based signal recovery problems are often relaxed to a convex surrogate such as $l_{1}$-norm minimization, which creates a unimodal optimization problem that minimizes the mean absolute error and practically converges to a maximally sparse solution. Rewriting the underlying problem as the $l_{1}$-norm minimization problem [20],

$$
\min \|\mathbf{u}\|_{1} \quad \text { subject to } \quad \mathbf{y}=\boldsymbol{\Phi} \mathbf{u},
$$

which can be solved using the sparse signal reconstruction algorithms such as Least Absolute Shrinkage and Selection Operator (LASSO) [21], Orthogonal Matching Pursuit (OMP) [22], and Compressive Sampling Matching Pursuit (CoSaMP) [23].

In this study, we use standard maximum likelihood search for a single reader scenario as we focus on the localization of a single reader.

\section{B. Precise RFID Reader Localization with Multipath Propagation}

Multipath propagation, due to the presence of different reflecting and scattering objects in the propagation scene, can be another major source of error in RFID reader localization. Rapid fluctuations in signal strength due to fading result in inconsistent and inaccurate range estimates. For precise localization, it is imperative to account for all significant multipath components. In this section, we consider the case where the reflector is a flat surface with a known position. Such situations arise, for example, when the reader is mounted on a vehicle in a warehouse with reflective surface, whereas other reflectors are distant from the vehicle. In such a situation, signal transmitted from the reader follows at least two distinct paths: a direct path (reader-tag-reader) and a reflection path (reader-wall-tag-reader) as illustrated in figure 2. Signals can also propagate along multiple reflection paths; nonetheless, they are severely attenuated, and hence, can be considered negligible in the RSSI measurement. Along the direct path, the distance between the $i$ th tag, located at $\left(x_{i}, y_{i}, 0\right)$ and the reader, at an unknown location $\left(x_{k}, y_{k}, h\right)$, is defined in equation (11). Similarly, the propagation distance between the $i$ th tag and the reader, along the reflection path is defined as

$$
d_{r, i}^{k}=\sqrt{\left(x_{i}-x_{k}\right)^{2}+\left(y_{i}+y_{k}\right)^{2}+h^{2}} .
$$




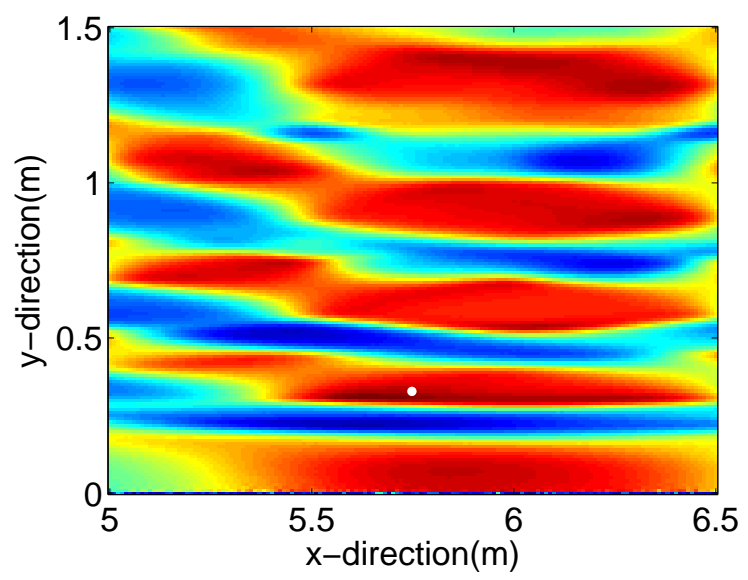

Figure 3. Correlation result showing the effect of strong reflection

Corresponding path difference between the direct LOS path and the reflection path is

$$
\Delta d_{i}^{k}=d_{i}^{k}-d_{r, i}^{k}
$$

and the equivalent phase difference is

$$
\Delta \theta_{i}^{k}=\frac{2 \pi \Delta d_{i}^{k}}{\lambda}
$$

Following the ground reflection (two-ray) model, the total signal power reaching the $i$ th tag is calculated as [24]

$$
P_{i, \mathrm{tag}}^{k}=P_{T X}\left(\frac{\lambda}{4 \pi}\right)^{2}\left|\frac{\sqrt{G_{\mathrm{LOS}}}}{d_{i}^{k}}+\frac{\Gamma \sqrt{G_{\mathrm{RFL}}} \exp \left(-\mathrm{j} \Delta \theta_{i}^{k}\right)}{d_{r, i}^{k}}\right|^{2},
$$

where $G_{L O S}$ is the product of the reader and tag antenna field pattern in the LOS direction and $G_{R F L}$ is the product of reader and antenna field patterns in the direction of the point of reflection. Consequently, the total instantaneous power reflected back to the reader from the $i$ th tag can be expressed as

$$
P r_{i}^{k}=P_{i, \mathrm{tag}}^{k}\left(\frac{\lambda}{4 \pi}\right)^{2}\left|\frac{\sqrt{G_{\mathrm{LOS}}}}{d_{i}^{k}}+\frac{\Gamma \sqrt{G_{\mathrm{RFL}}} \exp \left(-\mathrm{j} \Delta \theta_{i}^{k}\right)}{d_{r, i}^{k}}\right|^{2}=P_{T X}\left(\frac{\lambda}{4 \pi}\right)^{4}\left|\frac{\sqrt{G_{\mathrm{LOS}}}}{d_{i}^{k}}+\frac{\Gamma \sqrt{G_{\mathrm{RFL}}} \exp \left(-\mathrm{j} \Delta \theta_{i}^{k}\right)}{d_{r, i}^{k}}\right|^{4} .
$$

In order to incorporate the effect of multiple path propagation, the dictionary matrix $\mathbf{\Phi}$ is defined such that its $k$ th column stacks the power received from the $i$ th tag as defined in equation (20).

After compensating for the impairments due to non-isotropic radiation pattern and multipath propagation, the underlying problem can be solved using the ML estimation or one of the many $l_{1}$-norm minimization methods mentioned in the previous section.

Spatial resolution of the search grid imposes a limit on the achievable accuracy of estimation. Larger regression matrix, $\boldsymbol{\Phi}$, corresponding to a finer search grid, results in more accurate estimate. The issue of grid resolution is critical in presence of strong multipath, particularly when the distance between the reader antenna and the reflecting surface is small. The multipath signal components, propagating along the direct path and the reflection path, superpose with each other forming a quasi-periodic standing wave pattern, as shown in Figure 3. Under such conditions, a very dense spatial sampling, on the order of a small fraction of the wavelength, is required to achieve a robust solution. 


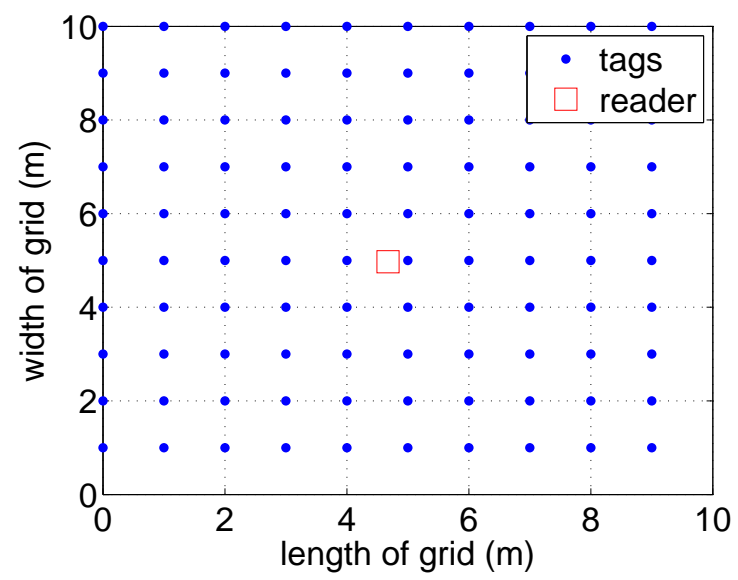

Figure 4. RFID grid configuration

\section{SIMULATION RESULTS}

We consider a typical indoor passive RFID deployment with 100 uniformly distributed tags across a square search area of $100 \mathrm{~m}^{2}$. In order to analyze the problem in 2-D, the reader antenna is assumed to be located at a constant height of $1 \mathrm{~m}$ above the ground while all the tags are assumed to be located on the ground. In the multipath propagation environment, we assume a reflecting surface along one of the boundaries of the rectangular grid at $y=0$. Figure 4 illustrates the top-view of the RFID grid configuration and tags-reader distribution in relation to the reflecting surface assumed for the simulation.

In this study, we assume a sinusoidal antenna radiation pattern for the reader antenna in order to analyze performance of the proposed method for non-isotropic radiation pattern, such that

$$
R(\theta)=\sin ^{2}(\theta), \quad \frac{\pi}{2} \leq \theta \leq \pi,
$$

where $\theta$ represents the elevation angle in radians measured between the reader and a tag under consideration. Tag antennas are assumed to have isotropic radiation pattern. The carrier frequency is assumed to be $915 \mathrm{MHz}$, corresponding to the center of the $902-928 \mathrm{MHz}$ band commercially used by the RFID transmitters. The ratio between the energy of the backscattered signal bit and the additive noise spectrum is denoted as $E_{b} / N_{0}$. The noise power is determined such that $E_{b} / N_{0}$ equals to $5 \mathrm{~dB}$ when a tag is $5 \mathrm{~m}$ away from the reader. Assuming that a 96-bit frame of the backscattered signal is used for localization, the corresponding processing gain is used to calculate $E_{b} / N_{0}$.

The root-mean-square-error (RMSE) of the reader position estimate is used as a performance metric to evaluate the impacts of the propagation impairments, separately and in tandem, on the RFID reader localization. Along the $x$-direction, 50 random numbers are drawn from a uniform distribution, and these values are used for each of the 5 arbitrarily selected reader-wall distance. Reflection coefficient of the wall is assumed to be -1 for this simulation, and accordingly, a dense search grid is defined by choosing a grid resolution of $5 \mathrm{~cm}(\approx \lambda / 6)$.

First, we evaluate performance of the proposed algorithm in two extreme environments. First, an ideal propagation environment, i.e., no multipath and isotropic radiation pattern, is assumed. Next, we observe performance of the proposed method under the combined effect of both impairments. These two sets of RMSE values provide baseline for assessing performance of the proposed method in different levels of impairments. In order to analyze the separate effects of directional antenna and multipath propagation on positioning accuracy, the experiment is repeated for two cases of impairments: directional antenna only and multipath only. As shown in Figure 5, the ML search yields highly accurate estimations, for a sufficiently high SNR and no propagation impairments, whereas the estimation error is on the order of a meter when both the impairments are in effect. The errors due to multipath and directional antenna pattern are also significantly high. These simulation results indicate a need for mechanism to compensate for the propagation impairments to achieve a precise position 


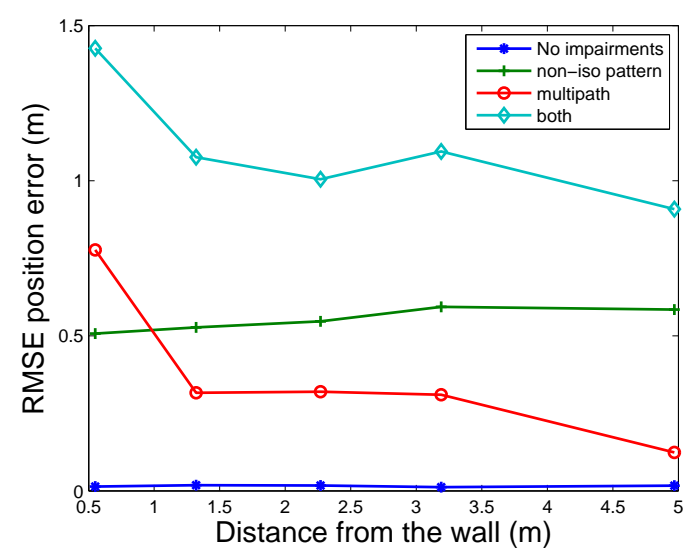

Figure 5. Effect of propagation impairments

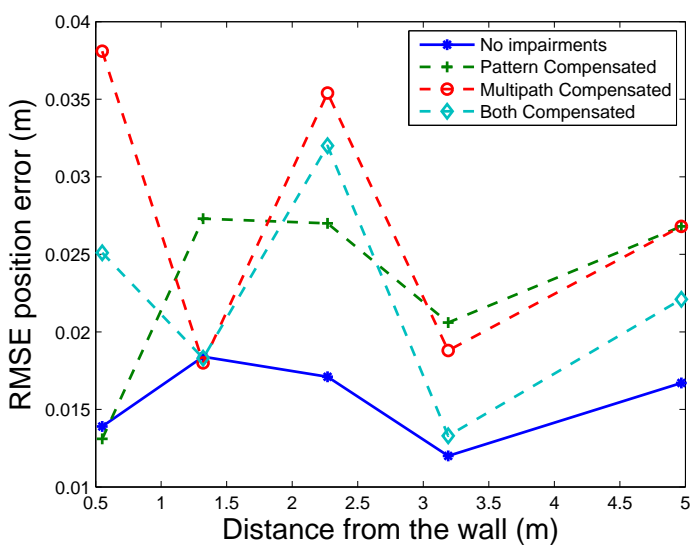

Figure 6. RMSE of maximum likelihood position estimate after compensation

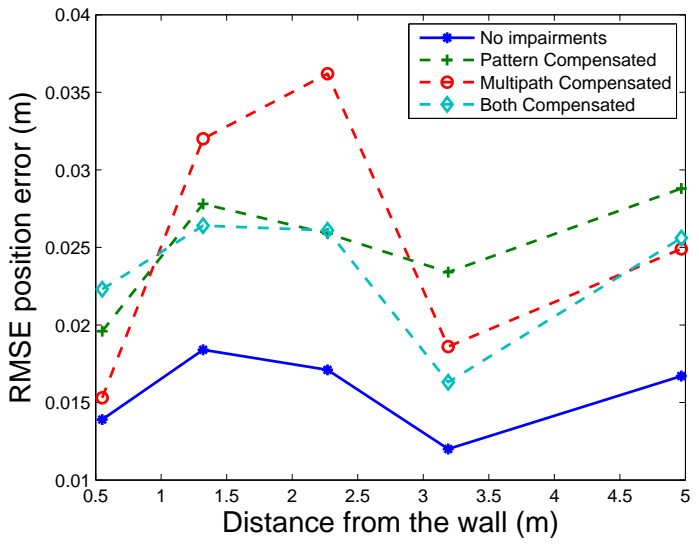

Figure 7. RMSE of position estimate using LASSO after compensation 
estimation of the RFID reader. Next, we evaluate the ability of the proposed method based on sparse signal recovery to compensation for these propagation effects and achieve a precise localization of the RFID reader.

We compare the compensated results in three different cases of impairments, i.e., multipath propagation only, non-isotropic antenna pattern only, and both; the respective result obtained using the ML search is presented in Figure 6. It can be observed that the position estimation error is reduced to a satisfactory level in all three cases. This verifies the ability of the proposed method to effectively compensate for the propagation impairments. Similar performance is obtained using LASSO with regularization parameter set to 0.1, as shown in Figure 7 .

As can be observed in the simulation results, the RMSE of reader position estimates closely approaches the ideal propagation conditions after compensation. However, in practical situations, the observation vector is likely to be affected by measurement error, which may affect the performance of the proposed method.

\section{CONCLUSIONS}

In this paper, we have developed a method for localizing an RFID reader based on sparse signal recovery. The proposed technique is based on multilateration exploiting RSSI measurements for range estimation. Existing methods are not equipped to decouple the effects of channel impairments, multipath propagation and nonisotropic antenna patterns, on the RSSI measurements. As a result, they fail to provide robust location estimation under such challenging propagation conditions. The proposed method compensates for both impairments and yields a highly precise location estimation. Simulation results are presented to verify the robustness of the proposed method in comparison to the conventional multilateration based RFID localization method.

\section{REFERENCES}

[1] Y. D. Zhang, M. G. Amin, and S. Kaushik, "Localization and tracking of passive RFID tags based on direction estimation," International Journal of Antennas and Propagation, vol. 2007, Dec. 2007.

[2] Y. D. Zhang, X. Li, and M. G. Amin, "Array processing for RFID tag localization exploiting multi-frequency signals," in Proceedings of SPIE Wireless Sensing and Processing IV, vol. 7349, Orlando, FL, Apr. 2009.

[3] Y. Zhang, X. Li, and M. G. Amin, "Principles and techniques of RFID positioning," in RFID Systems Research Trends and Challenges, M. Bolić, D. Simplot-Ryl, I. Stojmenović, eds., Wiley, pp. 389-412, 2010.

[4] G. Marrocco, E. Di Giampaolo, and R. Aliberti, "Estimation of UHF RFID Reading Regions in Real Environments," IEEE Antennas and Propagation Magazine, vol. 51, no. 6, pp. 44-57, Dec. 2009.

[5] F. Izquierdo, M. Ciurana, F. Barcelo, J. Paradells, and E. Zola, "Performance evaluation of a TOA-based trilateration method to locate terminals in WLAN," in Proceedings of International Symposium on Wireless Pervasive Computing, Phuket, Thailand, Jan. 2006.

[6] T. Wang, "Novel sensor location scheme using time-of-arrival estimates," IET Signal Processing, vol. 6, no. 1, pp. 8-13, Feb. 2012.

[7] C. Hekimian-Williams, B. Grant, L. Xiuwen, Z. Zhang, and P. Kumar, "Accurate localization of RFID tags using phase difference," in Proceedings of IEEE International Conference on RFID, Orlando, FL, Apr. 2010.

[8] X. Li, Y. Zhang, and M. G. Amin, "Multifrequency-based range estimation of RFID tags," in Proceedings of IEEE International Conference on RFID, Orlando, FL, April 2009.

[9] Y. Zhang and M. G. Amin, "Localization and tracking of passive RFID tags," in Proceedings of SPIE Wireless Sensing and Processing, Orlando, FL, April 2006.

[10] A. H. Sayed, A. Tarughat, and N. Khajehnouri, "Network-based wireless location: challenges faced in developing techniques for accurate wireless location information," IEEE Signal Processing Magazine, vol. 22, no. 4, pp. 24-40, July 2005.

[11] Z. Xiong, F. Sottile, M. A. Spirito, and R. Garello, "Hybrid indoor positioning approaches based on WSN and RFID," in Proceedings of IFIP International Conference on New Technologies, Mobility and Security (NTMS), Paris, France, Feb. 2011.

[12] A. Milella, P. Vanadia, G. Cicirelli, and A. Distante, "RFID-based environment mapping for autonomous mobile robot applications," in Proceedings of IEEE/ASME International Conference on Advanced Intelligent Mechatronics, Zurich, Switzerland, Sept. 2007.

[13] J. Song, C. T. Haas, and C. H. Caldas, "A proximity-based method for locating RFID tagged objects," Advanced Engineering Informatics, vol. 21, no. 4, pp. 367-376, Oct. 2007.

[14] M. Bouet and A. L. Dos Santos, "RFID tags: Positioning principles and localization techniques," in Proceedings of IFIP Wireless Days Conference, Dubai, UAE, Nov. 2008.

[15] Y. Zhang, K. Yemelyanov, X. Li, and M. G. Amin, "Effect of metallic objects and liquid supplies on RFID links," in Proceedings of IEEE AP-S International Symposium on Antennas and Propagation and USNC/URSI National Radio Science Meeting, Charleston, SC, June 2009. 
[16] J. Wang, M. G. Amin, and Y. D. Zhang, "Signal and array processing techniques for RFID readers," in Proceedings of SPIE Symposium on Wireless Sensing and Processing, vol. 6248, Orlando, FL, Apr. 2006.

[17] D. M. Dobkin, The RF in RFID: Passive UHF RFID in Practice, Burlington, MA: Newnes, 2008.

[18] Y. Zhao, N. Patwari, P. Agrawal, and M. G. Rabbat, "Directed by directionality: Benefiting from the gain pattern of active RFID badges," IEEE Transactions on Mobile Computing, vol. 11, no. 5, pp. 865-877, May 2012.

[19] P. Asadzadeh, L. Kulik, and E. Tanin, "Gesture recognition using RFID technology," Personal Ubiquitous Computing, vol. 16, no. 3, pp. 225-234, Mar. 2012.

[20] D. L. Donoho and Y. Tsaig, "Fast solution of $l_{1}$-norm minimization problems when the solution may be sparse," IEEE Transactions on Information Theory, vol. 54, no. 11, pp. 4789-4812, Nov. 2008.

[21] D. Angelosante and G. B. Giannakis, "RLS-weighted Lasso for adaptive estimation of sparse signals," in Proceedings of IEEE Acoustics, Speech and Signal Processing (ICASSP), pp. 3245-3248, Taipei, Taiwan, Apr. 2009.

[22] J. A. Tropp and A. C. Gilbert, "Signal recovery from random measurements via orthogonal matching pursuit," IEEE Transactions on Information Theory, vol. 53, no. 12, pp. 4655-4666, Dec. 2007.

[23] D. Needell and J. A. Tropp, "CoSaMP: Iterative signal recovery from incomplete and inaccurate samples," Applied and Computational Harmonic Analysis, vol. 26, pp. 301-321, 2008.

[24] T. S. Rappaport, Wireless Communications: Principles and Practice, Upper Saddle River, NJ: Prentice Hall PTR, 2002 . 